\title{
Outage probability analysis of dual energy harvesting relay network over rayleigh fading channel using SC and MRC technique
}

\author{
Van-Duc Phan ${ }^{1}$, Tan N. Nguyen², Minh Tran³, Tran Thanh Trang ${ }^{4}$ \\ ${ }^{1}$ Center of Excellence for Automation and Precision Mechanical Engineering, Nguyen Tat Thanh University, Vietnam \\ ${ }^{2}$ Wireless Communications Research Group, Faculty of Electrical and Electronics Engineering, \\ Ton Duc Thang University, Vietnam \\ ${ }^{3}$ Optoelectronics Research Group, Faculty of Electrical and Electronics Engineering, \\ Ton Duc Thang University, Vietnam \\ ${ }^{4}$ Faculty of Engineering and Technology, Van Hien University, Vietnam
}

\begin{tabular}{|c|c|}
\hline Article Info & ABSTRACT \\
\hline Article history: & In this paper, the system model of dual-energy harvesting relay network over \\
\hline Received Jan 11, 2019 & $\begin{array}{l}\text { Rayleigh fading channel and the comparison between Selecting Combining } \\
\text { (SC) and Maximal Ratio Combining (MRC) technique cases are proposed }\end{array}$ \\
\hline Revised Apr 11, 2019 & and investigated. The closed-form expression of the outage probability for \\
\hline Accepted May 9, 2019 & $\begin{array}{l}\text { the SC case and the integral-form expression of the outage probability for } \\
\text { MRC case is derived. Moreover, the influence of the main parameters on the }\end{array}$ \\
\hline Keywords: & $\begin{array}{l}\text { system performance is demonstrated entirely by the Monte Carlo simulation. } \\
\text { From the results, we can see that all simulation and analytical results match } \\
\text { well with each other. }\end{array}$ \\
\hline
\end{tabular}

Half-duplex (HD)

Maximal ratio combining

(MRC)

Relaying network

Selection combining (SC)

Copyright (C) 2019 Institute of Advanced Engineering and Science. All rights reserved.

\section{Corresponding Author:}

Tan N. Nguyen,

Wireless Communications Research Group,

Faculty of Electrical and Electronics Engineering,

Ton Duc Thang University, Ho Chi Minh City, Vietnam.

Email: nguyennhattan@tdtu.edu.vn

\section{INTRODUCTION}

Nowadays, the fifth generation $(5 \mathrm{G})$ network technology is the best solution for the near future communication network. However, increase the energy efficiency of wireless communication networks is the critical problem, on which are strongly depended the economic and ecological aspects of 5G networks. For this target, two solutions are proposed and demonstrated. Firstly, the overall energy consumption of future 5G network shall not exceed 10 percent of the current usage. Secondly, much longer battery life for mobile devices is expected [1-7]. Significant technological steps would have to be taken shortly for this goal to become a reality. Several candidate solutions have been proposed lately to meet the goals above. Technologies based on radio frequency $(\mathrm{RF})$ energy harvesting $(\mathrm{EH})$ and transfer have recently been gaining momentum. With these approaches, future wireless devices would have the capability of harvesting energy from signals emitted either by ambient or dedicated sources [1-7]. In the last few years, radio frequency (RF) energy harvesting $(\mathrm{EH})$ as one of the promising techniques, has received much attention, since it can provide unlimited power to the sensor nodes which scavenge energy from the environment (i.e., solar, wind, etc.). Among these, RF energy radiated by ambient transmitters is almost ubiquitous, which can be harvested more effectively from wireless RF signals. Since RF signal can carry energy and information simultaneously, energy harvesting $(\mathrm{EH})$ and simultaneous wireless information and power transfer (SWIPT) are becoming a 
more and more promising research direction [1-7]. In the last few years, the system performance of the energy harvesting relay network has been considered in many studies. [8] investigated the full-duplex energy harvesting relay network with simultaneous energy harvesting and information transmission. Moreover, the development of cooperative protocols for energy harvesting relay network is deeply studied in [9-10]. Furthermore, [11-12] proposed a "harvest-then-transmit" protocol for a multi-user relay network.

In this work, the outage probability analysis of dual-energy harvesting relay network over Rayleigh fading channel using Selection Combining (SC) and Maximal Ratio Combining (MRC) technique is presented and investigated. For details on this analysis, the energy, and information are transferred from the source to the relay nodes, and all channels are considered as the Rayleigh fading channels. The main contributions of the paper are summarized as follows:

a) The system model of dual-energy harvesting relay network over Rayleigh fading channel and the comparison between Selection Combining (SC) and Maximal Ratio Combining (MRC) technique cases are proposed and investigated.

b) The closed-form expression of the outage probability for the SC case and the integral-form expression of the outage probability for the MRC case is derived.

c) The influence of the main parameters on the system performance is demonstrated entirely by the Monte Carlo simulation.

The structure of this paper is proposed as follows. Sections 2 presents the system model of the relaying network. Sections 3 derives the system performance of the model system. Section 4 provides numerical results and some discussions. Finally, Section 5 concludes the paper.

\section{SYSTEM MODEL}

In this section, Figure 1 plots the system model of the dual energy harvesting $(\mathrm{EH})$ relaying network over a Rayleigh fading channel with one Source $(S)$, one Destination (D) and two helping Relay $\left(R_{1}\right.$ and $\left.R_{2}\right)$. Moreover, Figure 2 illustrates the EH and Information Transmission (IT) phases of the model system with the block time $T$. In the first interval time $(\alpha \mathrm{T})$, the $\mathrm{R}_{1}$ and $\mathrm{R}_{2}$ harvest energy from the source signal, where $\alpha$ is the time switching factor $\alpha \in(0,1)$. After that, in the $(1-\alpha) \mathrm{T} / 2$ interval time, The $\mathrm{S}$ transfers information to the $R_{1}$ and $R_{2}$. In the remaining interval time $(1-\alpha) T / 2$, the $R_{1}$ and $R_{2}$ node transfer information to the $D$. All the fading channels from $\mathrm{S}$ to $\mathrm{R}$ and $\mathrm{R}$ to $\mathrm{D}$ are proposed as the Rayleigh fading channels. More details of the analytical mathematical model of the outage probability and throughput of the system model is presented and analyzed in the following sections [13-25].

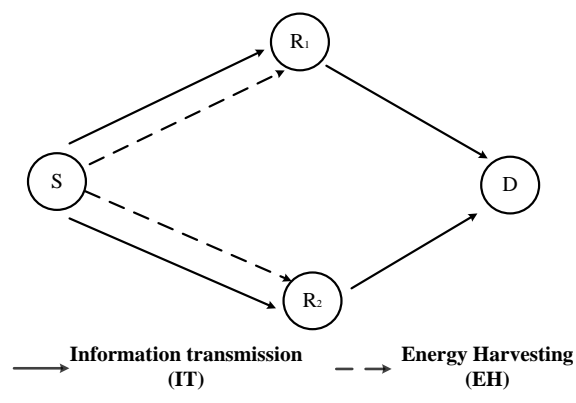

Figure1. System model

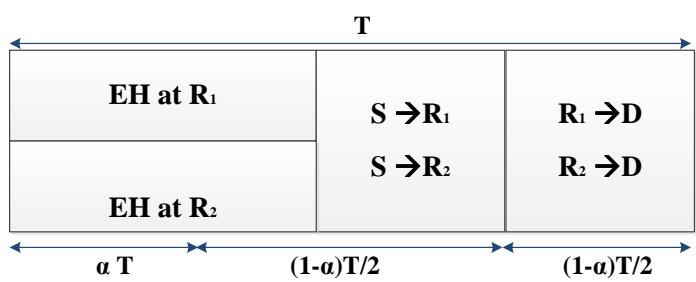

Figure 2. The power splitting protocol

\subsection{Energy Harvesting Phase}

The received signal at $R_{1}$ can be expressed as

$$
y_{r_{1}}=h_{s r_{1}} x_{s}+n_{r_{1}}
$$

Where $x_{s}$ is the transmit signal at the source and $\mathrm{E}\left\{\left|x_{s}\right|^{2}\right\}=P_{s}, \mathrm{E}\{\bullet\}:$ expectation operator, $\mathrm{P}_{\mathrm{s}}$ is transmitting power of the source, $n_{r_{1}}$ : the additive white Gaussian noise (AWGN) at the relay $\mathrm{R}_{1}$ with zeromean and variance $\mathrm{N}_{0}$. 
The harvested energy at $R_{1}$ can be given by

$$
E_{r_{1}}=\alpha T \eta P_{s}\left|h_{s r_{1}}\right|^{2}
$$

From (2), the average transmit power at $\mathrm{R}_{1}$ can be obtained as

$$
P_{r_{1}}=\frac{E_{r_{1}}}{(1-\alpha) T / 2}=\mu P_{s}\left|h_{s r_{1}}\right|^{2}
$$

Where we denote $\mu=\frac{2 \eta \alpha}{1-\alpha}, 0<\eta \leq 1$ is the energy conversion efficiency and $0<\alpha<1$ is the time switching factor.

Similarity, the received signal at $\mathrm{R}_{2}$ can be expressed as

$$
y_{r_{2}}=h_{s r_{2}} x_{s}+n_{r_{2}}
$$

Where $n_{r_{2}}$ is the AWGN at the relay $\mathrm{R}_{2}$ with zero-mean and variance $\mathrm{N}_{0}$.

The average transmit power at $\mathrm{R}_{2}$ can be claimed as

$$
P_{r_{2}}=\frac{E_{r_{2}}}{(1-\alpha) T / 2}=\mu P_{s}\left|h_{s r_{2}}\right|^{2}
$$

\subsection{Information transmission phase}

The received signal at $D$ from the transmitted signal of $R_{1}$ and $R_{2}$ can be expressed as the followings, respectively.

$$
\begin{aligned}
& y_{d_{1}}=h_{r_{1} d} x_{r_{1}}+n_{d} \\
& y_{d_{2}}=h_{r_{2} d} x_{r_{2}}+n_{d}
\end{aligned}
$$

Where $x_{r_{1}}, x_{r_{2}}$ are the transmit signal at the relay $\mathrm{R}_{1}$ and $\mathrm{R}_{2}$, respectively. $\mathrm{E}\left\{\left|x_{r_{1}}\right|^{2}\right\}=P_{r_{1}}$ and $n_{d}$ is the AWGN at the destination with zero-mean and variance $\mathrm{N}_{0}$.

After received the signal from $\mathrm{S}$, the signal at the relay $\mathrm{R}_{1}$ will be amplified by amplifying factor as

$$
\beta_{1}=\frac{x_{r_{1}}}{y_{r_{1}}}=\sqrt{\frac{P_{r_{1}}}{P_{s}\left|h_{s r_{1}}\right|^{2}+N_{0}}}
$$

Substituting (1) and (7) into (6), the received signal at the destination from $\mathrm{R}_{1}$ can be obtained as

$$
\begin{aligned}
& y_{d_{1}}=h_{r_{1} d} \beta_{1} y_{r_{1}}+n_{d}=h_{r_{1} d} \beta_{1}\left[h_{s r_{1}} x_{s}+n_{r_{1}}\right]+n_{d}
\end{aligned}
$$

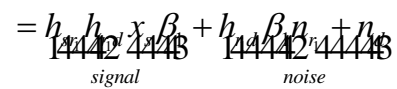

The end to end signal to noise (SNR) ratio of $\mathrm{S}-\mathrm{R}_{1}-\mathrm{D}$ link can be claimed as

$$
\gamma_{1}=\frac{\mathrm{E}\left\{\mid \text { signal }\left.\right|^{2}\right\}}{\mathrm{E}\left\{\mid \text { noise }\left.\right|^{2}\right\}}=\frac{\left|h_{s r_{1}}\right|^{2}\left|h_{r_{1} d}\right|^{2} P_{s} \beta_{1}^{2}}{\left|h_{r_{1} d}\right|^{2} \beta_{1}^{2} N_{0}+N_{0}}
$$

Using the fact that $\mathrm{N}_{0}<<\mathrm{P}_{\mathrm{r} 1}$, then, substituting (3) and (7) into (9), the (9) can be rewritten as 


$$
\gamma_{1} \approx \frac{\mu\left|h_{s r_{1}}\right|^{2}\left|h_{r_{d} d}\right|^{2} \Delta}{\mu\left|h_{r_{1} d}\right|^{2}+1}
$$

Where we denote $\Delta=\frac{P_{s}}{N_{0}}$

Similarity, the SNR of S- $\mathrm{R}_{2}$-D link can be obtained as

$$
\gamma_{2} \approx \frac{\mu\left|h_{s r_{2}}\right|^{2}\left|h_{r_{2} d}\right|^{2} \Delta}{\mu\left|h_{r_{2} d}\right|^{2}+1}
$$

\section{THE SYSTEM PERFORMANCE}

\subsection{Selection Combining (SC)}

In this section, we consider the diversity technique $\mathrm{SC}$ at the receiver. In this technique, the destination will choose the best SNR between $S-R_{1}-D$ and $S-R_{2}-D$ link

Hence, the end to end SNR can be given by

$$
\gamma_{e 2 e}^{S C}=\max \left(\gamma_{1}, \gamma_{2}\right)
$$

Substituting (10) and (11) into (12), finally, the end to end SNR at D can be rewritten as

$$
\gamma_{e 2 e}^{S C}=\max \left(\frac{\mu\left|h_{s r_{1}}\right|^{2}\left|h_{r_{d} d}\right|^{2} \Delta}{\mu\left|h_{r_{1} d}\right|^{2}+1}, \frac{\mu\left|h_{s r_{2}}\right|^{2}\left|h_{r_{d} d}\right|^{2} \Delta}{\mu\left|h_{r_{2} d}\right|^{2}+1}\right)
$$

Outage probability OP) analysis

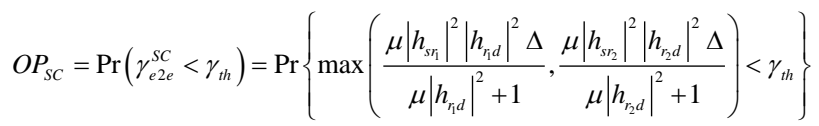

$$
\begin{aligned}
& =\operatorname{Pr}\left(\frac{\mu\left|h_{s i n_{1}}\right|^{2}\left|h_{r_{d}}\right|^{2} \Delta}{\mu\left|h_{r_{d}}\right|^{2}+1}<\gamma_{t h}\right) \times \operatorname{Pr}\left(\frac{\mu\left|h_{s s^{2}}\right|^{2}\left|h_{r^{d}}\right|^{2} \Delta}{\mu\left|h_{r_{2} d}\right|^{2}+1}<\gamma_{t h}\right) \\
& =P_{1} \times P_{2}
\end{aligned}
$$

Where $\gamma_{t h}=2^{2 R}-1$ is the threshold and $\mathrm{R}$ is the target rate.

We denote as

$$
\begin{aligned}
P_{1} & =\operatorname{Pr}\left(\frac{\mu\left|h_{s i}\right|^{2}\left|h_{r d}\right|^{2} \Delta}{\mu\left|h_{r d}\right|^{2}+1}<\gamma_{t h}\right)=\operatorname{Pr}\left[\left|h_{s i}\right|^{2}<\frac{\gamma_{t h}}{\Delta}+\frac{\gamma_{t h}}{\mu \Delta\left|h_{r d}\right|^{2}}\right] \\
& =\int_{0}^{\infty} F_{\left|h_{h l}\right|^{2}}\left(\frac{\gamma_{t h}}{\Delta}+\frac{\gamma_{t h}}{\mu \Delta\left|h_{r d}\right|^{2}}\left|h_{s i}\right|^{2}\right) f_{\left|h_{r d}\right|^{2}}\left(\left|h_{r d}\right|^{2}\right) d\left(\left|h_{r d}\right|^{2}\right) \\
& =\int_{0}^{\infty} F_{X}\left(\frac{\gamma_{t h}}{\Delta}+\frac{\gamma_{t h}}{\mu \Delta Y} \mid X\right) f_{Y}(Y) d Y
\end{aligned}
$$

Where we denote $X=\left|h_{s r_{1}}\right|^{2}, Y=\left|h_{r_{i}}\right|^{2}$.

Then the (15) can be obtained as 


$$
\begin{aligned}
P_{1} & =1-\lambda_{r_{1} d} \int_{0}^{\infty} \exp \left[-\lambda_{s r_{1}}\left(\frac{\gamma_{t h}}{\Delta}+\frac{\gamma_{t h}}{\mu \Delta Y}\right)\right] \times \exp \left(-\lambda_{r_{1} d} Y\right) d Y \\
& =1-\lambda_{r_{1} d} \exp \left(-\frac{\lambda_{s r_{1}} \gamma_{t h}}{\Delta}\right) \int_{0}^{\infty} \exp \left(-\frac{\lambda_{s r_{1}} \gamma_{t h}}{\mu \Delta Y}\right) \times \exp \left(-\lambda_{r_{1} d} Y\right) d Y
\end{aligned}
$$

Where $\lambda_{s r_{1}}, \lambda_{r_{1} d}$ are the mean of the random variable $\left|h_{s r_{1}}\right|^{2},\left|h_{r_{1} d}\right|^{2}$, respectively.

Apply equation $(3.324,1)$ of the table of integral [26], from(16) can be reformulated as

$$
P_{1}=1-2 \exp \left(-\frac{\lambda_{s r_{1}} \gamma_{t h}}{\Delta}\right) \times \sqrt{\frac{\lambda_{s r_{1}} \lambda_{r_{1} d} \gamma_{t h}}{\mu \Delta}} \times K_{1}\left(2 \sqrt{\frac{\lambda_{s r_{1}} \lambda_{r_{1} d} \gamma_{t h}}{\mu \Delta}}\right)
$$

Where $K_{v}(\bullet)$ is the modified Bessel function of the second kind and $\mathrm{v}^{\text {th }}$ order.

Similarity, $\mathrm{P}_{1}, \mathrm{P}_{2}$ can be claimed as

$$
P_{2}=1-2 \exp \left(-\frac{\lambda_{s r_{2}} \gamma_{t h}}{\Delta}\right) \times \sqrt{\frac{\lambda_{s r_{2}} \lambda_{r_{2} d} \gamma_{t h}}{\mu \Delta}} \times K_{1}\left(2 \sqrt{\frac{\lambda_{s r_{2}} \lambda_{r_{2} d} \gamma_{t h}}{\mu \Delta}}\right)
$$
the following

Finally, substituting (17), (18) into (14), the OP by using SC technique can be obtained as

$$
\begin{aligned}
O P_{S C} & =\left\{1-2 \exp \left(-\frac{\lambda_{s r_{1}} \gamma_{t h}}{\Delta}\right) \times \sqrt{\frac{\lambda_{s r_{1}} \lambda_{r_{1} d} \gamma_{t h}}{\mu \Delta}} \times K_{1}\left(2 \sqrt{\frac{\lambda_{s r_{1}} \lambda_{r_{1} d} \gamma_{t h}}{\mu \Delta}}\right)\right\} \\
& \times\left\{1-2 \exp \left(-\frac{\lambda_{s r_{2}} \gamma_{t h}}{\Delta}\right) \times \sqrt{\frac{\lambda_{s r_{2}} \lambda_{r_{2} d} \gamma_{t h}}{\mu \Delta}} \times K_{1}\left(2 \sqrt{\frac{\lambda_{s r_{2}} \lambda_{r_{2} d} \gamma_{t h}}{\mu \Delta}}\right)\right\}
\end{aligned}
$$

\subsection{Maximal Ratio Combining (MRC)}

In this technique, the signal at the destination from $S-R_{1}-D$ and $S-R_{2}-D$ link will be incorporated. Hence, the end to end SNR at the destination can be expressed as

$$
\gamma_{e 2 e}^{M R C}=\gamma_{1}+\gamma_{2}=\frac{\mu\left|h_{s r_{1}}\right|^{2}\left|h_{r_{1} d}\right|^{2} \Delta}{\mu\left|h_{r_{1} d}\right|^{2}+1}+\frac{\mu\left|h_{s r_{2}}\right|^{2}\left|h_{r_{2} d}\right|^{2} \Delta}{\mu\left|h_{r_{2} d}\right|^{2}+1}
$$

Outage probability analysis

$$
\begin{aligned}
O P_{M R C} & =\operatorname{Pr}\left(\gamma_{e 2 e}^{M R C}<\gamma_{t h}\right)=\operatorname{Pr}\left(\gamma_{1}+\gamma_{2}<\gamma_{t h}\right) \\
& =\int_{0}^{\gamma_{t h}} f_{y}(y) d y \int_{0}^{\gamma_{t h}-y} f_{x}(x) d x \\
& =\int_{0}^{\gamma_{t h}}\left[F_{x}\left(\gamma_{t h}-y\right)-F_{x}(0)\right] f_{y}(y) d y
\end{aligned}
$$

Where we denote $x=\gamma_{1}, y=\gamma_{2}$, and $F_{x}\left(\gamma_{t h}\right)=\operatorname{Pr}\left(x<\gamma_{t h}\right)=\operatorname{Pr}\left(\gamma_{1}<\gamma_{t h}\right)$.

So, from (17), easily to observe that $F_{x}(0)=0$.

Hence, (21) can be rewritten as the following 


$$
O P_{M R C}=\int_{0}^{\gamma_{t h}} F_{x}\left(\gamma_{t h}-y\right) f_{y}(y) d y
$$

In order to calculate the OP in (22), we have to find $f_{y}(y)$. Based on the definition of probability density function (PDF) and cumulative distribution function (CDF), $f_{y}(y)$ can be obtained as

$$
f_{y}(y)=\frac{\partial F_{y}(y)}{\partial y}
$$

Using (18), we have

$$
F_{y}(y)=\operatorname{Pr}\left(\gamma_{2}<y\right)=1-2 \exp \left(-\frac{\lambda_{s r_{2}} y}{\Delta}\right) \times \sqrt{\frac{\lambda_{s r_{2}} \lambda_{r_{2} d} y}{\mu \Delta}} \times K_{1}\left(2 \sqrt{\frac{\lambda_{s r_{2}} \lambda_{r_{2} d} y}{\mu \Delta}}\right)
$$

Using the formula $\frac{d}{d x}\left(x^{v} K_{v}(x)\right)=-x^{v} K_{v-1}(x)$, from (23) can be reformulated as

$$
\begin{aligned}
f_{y}(y) & =\frac{2 \lambda_{s r_{2}}}{\Delta} \times \exp \left(-\frac{\lambda_{s r_{2}} y}{\Delta}\right) \times \sqrt{\frac{\lambda_{s r_{2}} \lambda_{r_{2} d} y}{\mu \Delta}} \times K_{1}\left(2 \sqrt{\frac{\lambda_{s r_{2}} \lambda_{r_{2} d} y}{\mu \Delta}}\right) \\
& +\frac{2 \lambda_{s r_{2}} \lambda_{r_{2} d}}{\mu \Delta} \times \exp \left(-\frac{\lambda_{s r_{2}} y}{\Delta}\right) \times K_{0}\left(2 \sqrt{\frac{\lambda_{s r_{2}} \lambda_{r_{2} d} y}{\mu \Delta}}\right)
\end{aligned}
$$

Combine (17) and substituting (25) into (22), the OP of the MRC technique can be claimed as

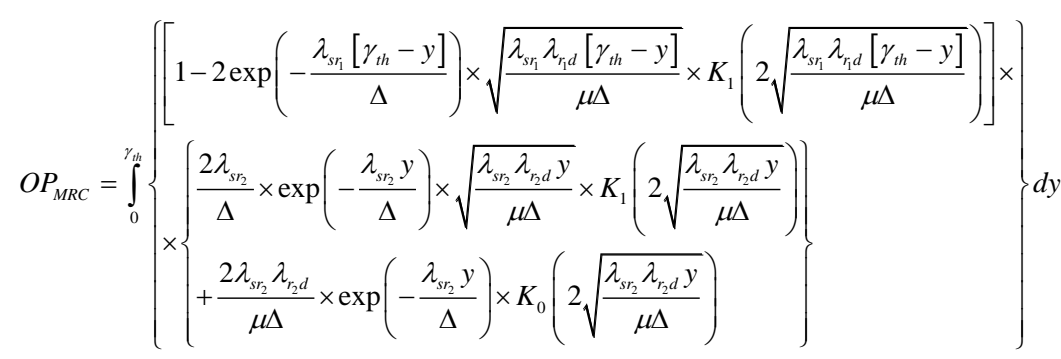

Throughput can be calculated as

$$
\tau_{S C o r M R C}=\left(1-O P_{S C o r M R C}\right) \times \frac{R(1-\alpha) T / 2}{T}=\left(1-O P_{S C o r M R C}\right) \times \frac{R(1-\alpha)}{2}
$$

\section{NUMERICAL RESULTS AND DISCUSSION}

In the simulation stage, we set that $\lambda_{s r_{1}}=0.5, \lambda_{r d_{1}}=1, \lambda_{s r_{2}}=1.5, \lambda_{s d_{2}}=2$. The influence of the energy efficiency coefficient $\eta$ on the OP and throughput of the model system are plotted in Figures 3 and 4 , respectively. As shown in these Figures, we can say that the OP falls, but the throughput rises with the rising $\eta$ from 0 to 1 . In this simulation step, we set $\mathrm{R}=1,2 \mathrm{bps} / \mathrm{Hz}, \Delta=5 \mathrm{~dB}$ and $\alpha=1$. It can be seen that the performance of the proposed system with SC technique is better than with the MRC technique and the simulation results are the same as the analytical results. In a similar way, the OP and throughput versus the time splitting factor $\alpha$ are shown in Figures 5 and 6 with $\eta=0.8, \Delta=1,5 \mathrm{~dB}$ and $\alpha=0.5$. As shown in Figure 5, the OP has a considerable decrease. The throughput rises when $\alpha$ varies from 0 to 0.5 and then fall crucially 
with $\alpha$ from 0.5 to 1 as plotted in Figure 6. The optimal value of the system throughput can be obtained at 0.5 of $\alpha$ as shown in Figure 6. Once again, the simulation and analytical results are the same.

Moreover, the influence of $\Delta$ on the Op and system throughput are illustrated in Figures 7 and 8 with $\alpha=0.5,0.85, \eta=0.8$ and $\Delta$ varies from $-5 \mathrm{~dB}$ to $25 \mathrm{~dB}$. Here, we can see that the OP significant decreases and throughput rises with rising $\Delta$. Also, the OP and system throughput versus $\mathrm{R}$ is drawn in Figures 9 and 10. It can be observed that the OP increases and throughput decrease significantly when the $\mathrm{R}$ varies from 0.5 to $2.5 \mathrm{bit} / \mathrm{s} / \mathrm{Hz}$. After that, the $\mathrm{OP}$ and throughput have slight changes when $\mathrm{R}$ increases from 2.5 to 5 $\mathrm{bit} / \mathrm{s} / \mathrm{Hz}$. In all the above Figs, the simulation and analytical results agree with each other.

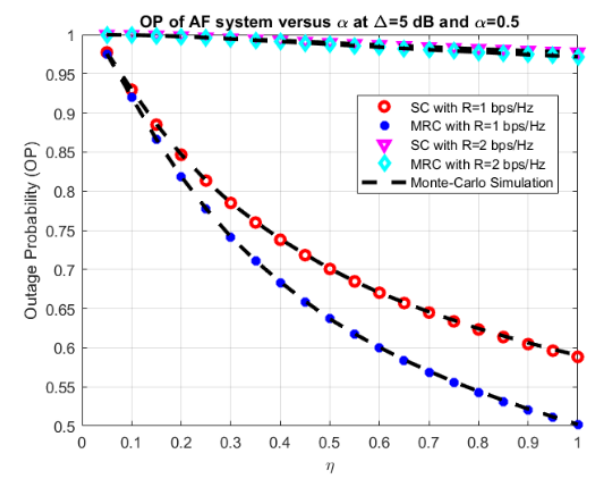

Figure 3. OP versus $\eta$

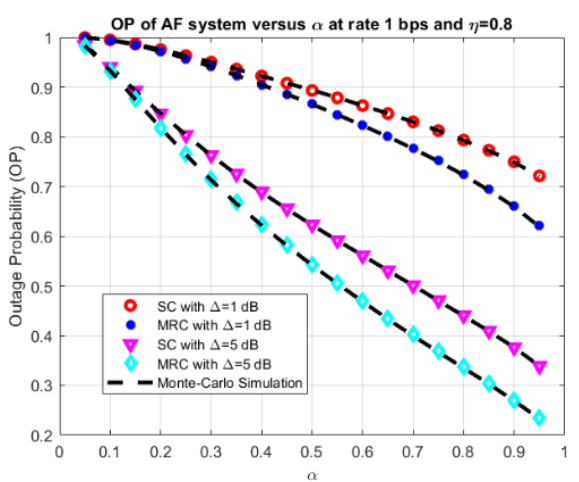

Figure 5. OP versus $\alpha$

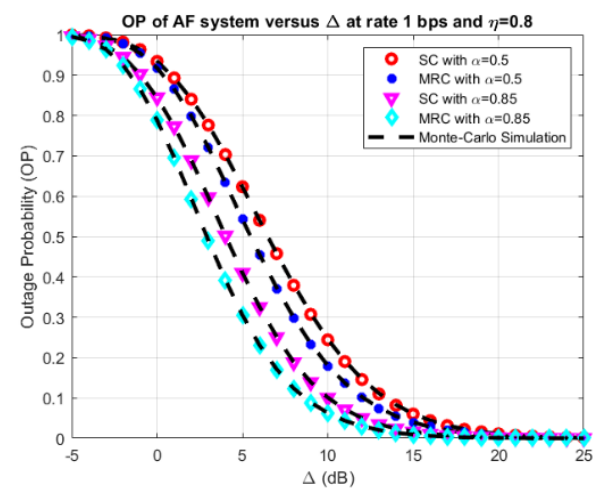

Figure 7. OP versus $\Delta$

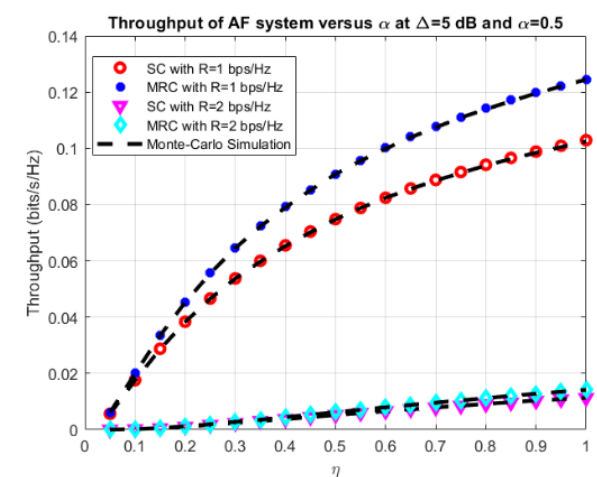

Figure 4. Throughput versus $\eta$

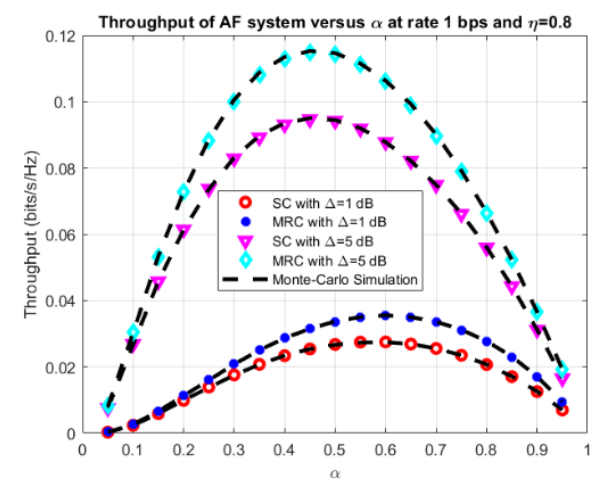

Figure 6. Throughput versus $\alpha$

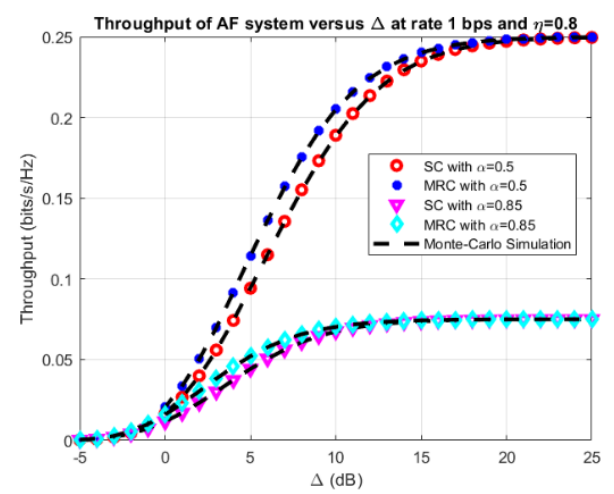

Figure 8 . Throughput versus $\Delta$ 


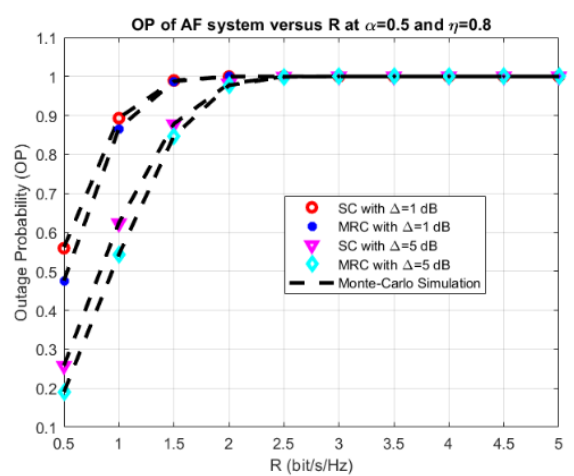

Figure 9. OP versus R

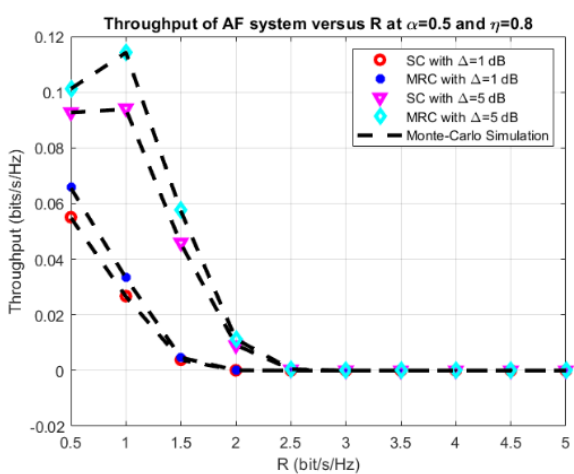

Figure 10. Throughput versus $\mathrm{R}$

\section{CONCLUSION}

In this paper, the system model of dual-energy harvesting relay network over Rayleigh fading channel and the comparison between SC and MRC techniques cases are proposed and investigated. The closed-form expression of the outage probability for the SC case and the integral-form expressions of the outage probability for MRC case is derived. Moreover, the influence of the main parameters on the system performance is demonstrated entirely by the Monte Carlo simulation. From the results, we can see that all simulation and analytical results match well with each other. This paper provides a novel recommendation for the communication relaying network shortly.

\section{REFERENCES}

[1] Q. Wu, G. Y. Li, W. Chen, D. W. K. Ng, and R. Schober, "An Overview of Sustainable Green 5G Networks," IEEE Wireless Communications, vol. 24, no. 4, pp. 72-80, 2017.

[2] D. Niyato, D. I. Kim, M. Maso, and Z. Han, "Wireless Powered Communication Networks: Research Directions and Technological Approaches," IEEE Wireless Communications, vol. 24, no. 6, pp. 88-97, 2017.

[3] T. D. P. Perera, D. N. K. Jayakody, S. K. Sharma, S. Chatzinotas, and J. Li, "Simultaneous Wireless Information and Power Transfer (SWIPT): Recent Advances and Future Challenges," IEEE Communications Surveys \&amp; Tutorials, vol. 20, no. 1, pp. 264-302, 2018.

[4] F. De Rango and M. Tropea, "Energy saving and load balancing in wireless ad hoc networks through ant-based routing", International Symposium on Performance Evaluation of Computer and Telecommunication Systems 2009, SPECTS 2009, pp. 117-124, 2009.

[5] Yu, H., Lee, H., \& Jeon, H., "What is 5G? Emerging 5G Mobile Services and Network Requirements," Sustainability, 9(10), 1848, 2017. doi:10.3390/su9101848.

[6] F. de Rango, P. Lonetti and S. Marano, "MEA-DSR: A multipath energy-aware routing protocol for wireless Ad Hoc Networks," IFIP International Federation for Information Processing, Vol. 265, pp. 215-225, 2008.

[7] Yao, Q., Huang, A., Shan, H., Quek, T. Q., \& Wang, W. (2016, 07). Adaptive harvest-then-cooperate: Delay-aware wireless powered communication networks. 2016 IEEE 17th International Workshop on Signal Processing Advances in Wireless Communications (SPAWC). doi:10.1109/spawc.2016.7536862.

[8] Gu, Y., Chen, H., Li, Y., \& Vucetic, B., "An adaptive transmission protocol for wireless-powered cooperative communications," 2015 IEEE International Conference on Communications (ICC), 2015, doi:10.1109/icc.2015.7248986.

[9] E. Chen, M. Xia, D. B. D. Costa, and S. Aissa, "Multi-Hop Cooperative Relaying With Energy Harvesting From Co-channel Interferences," IEEE Communications Letters, vol. 21, no. 5, pp. 1199-1202, 2017.

[10] Huang, K., \& Lau, V. K., "Enabling Wireless Power Transfer in Cellular Networks: Architecture, Modeling and Deployment," IEEE Transactions on Wireless Communications, 13(2), 902-912, 2014, doi:10.1109/twc.2013.122313.130727.

[11] Ma, Y., Chen, H., Lin, Z., Li, Y., \& Vucetic, B., "Distributed resource allocation for power beacon-assisted wireless-powered communications," 2015 IEEE International Conference on Communications (ICC). doi:10.1109/icc.2015.7248924.

[12] Park, J., Jeon, Y., \& Han, S., "Energy Beamforming for Wireless Power Transfer in MISO Heterogeneous Network With Power Beacon," IEEE Communications Letters, 21(5), 1163-1166, 2017. doi:10.1109/lcomm.2017.2654254.

[13] Chalise, B. K., Zhang, Y. D., \& Amin, M. G., "Energy harvesting in an OSTBC based amplify-and-forward MIMO relay system," 2012 IEEE International Conference on Acoustics, Speech and Signal Processing (ICASSP). doi:10.1109/icassp.2012.6288596 
[14] Tutuncuoglu, K., \& Yener, A., "Cooperative energy harvesting communications with relaying and energy sharing. 2013 IEEE Information Theory Workshop (ITW). doi:10.1109/itw.2013.6691280

[15] Krikidis, I., Timotheou, S., \& Sasaki, S., "RF Energy Transfer for Cooperative Networks: Data Relaying or Energy Harvesting?," IEEE Communications Letters, 16(11), 1772-1775, 2012. doi:10.1109/lcomm.2012.091712.121395

[16] Nishimoto, H., Kawahara, Y., \& Asami, T., "A prototype implementation of ambient RF energy harvesting wireless sensor networks,” 2010 IEEE Sensors. doi:10.1109/icsens.2010.5690588

[17] Zhang, R., \& Ho, C. K., "MIMO Broadcasting for Simultaneous Wireless Information and Power Transfer," IEEE Transactions on Wireless Communications, 12(5), 1989-2001, 2013. doi:10.1109/twc.2013.031813.120224

[18] Park, J., \& Clerckx, B., "Joint Wireless Information and Energy Transfer in a Two-User MIMO Interference Channel," IEEE Transactions on Wireless Communications, 12(8), 4210-4221, 2013. doi:10.1109/twc.2013.071913.130084

[19] Tan N. Nguyen, T.H.Q.Minh, Phuong T. Tran and Miroslav Voznak. Energy Harvesting over Rician Fading Channel: A Performance Analysis for Half-Duplex Bidirectional Sensor Networks under Hardware Impairments. Sensors, 18, 2018.

[20] Tan N. Nguyen, T.H.Q.Minh, Phuong T. Tran and Miroslav Voznak. Adaptive Energy Harvesting Relaying Protocol for Two-Way Half Duplex System Network over Rician Fading Channel. Wireless Communications and Mobile Computing, 2018.

[21] Tin, Phu Tran, Tran Hoang Quang Minh, Tan N. Nguyen, and Miroslav Voznak. "System Performance Analysis of Half-Duplex Relay Network over Rician Fading Channel," TELKOMNIKA (Telecommunication Computing Electronics and Control), 16, no. 1 (02, 2018): 189. doi:10.12928/telkomnika.v16i1.7491.

[22] Rashid, Tarique, Sunil Kumar, Akshay Verma, Prateek Raj Gautam, and Arvind Kumar. "Pm-EEMRP: Postural Movement Based Energy Efficient Multi-hop Routing Protocol for Intra Wireless Body Sensor Network (IntraWBSN)." TELKOMNIKA (Telecommunication Computing Electronics and Control), 16, no. 1 (02, 2018): 166. doi:10.12928/telkomnika.v16i1.7318.

[23] A. F. Morabito, "Power Synthesis of Mask-Constrained Shaped Beams Through Maximally-Sparse Planar Arrays," TELKOMNIKA (Telecommunication Computing Electronics and Control), vol. 14, n. 4, pp. 1217-1219, 2016.

[24] Mckay, Matthew R., Alex J. Grant, and Iain B. Collings. "Performance Analysis of MIMO-MRC in DoubleCorrelated Rayleigh Environments." IEEE Transactions on Communications 55, no. 3 (03 2007): 497-507. doi:10.1109/tcomm.2007.892450.

[25] Tran Hoang Quang Minh. "Hybrid Time-Power Switching Protocol of Energy Harvesting Bidirectional Relaying Network: Throughput and Ergodic Capacity Analysis." TELKOMNIKA (Telecommunication Computing Electronics and Control), 16, no. 5 (10, 2018): 189. http://dx.doi.org/10.12928/telkomnika.v16i5.9118.

[26] Table of Integrals, Series, and Products, 2015. 